\title{
A discrete evolutionary PSO based approach to the multiyear transmission expansion planning problem considering demand uncertainties
}

\author{
Manuel Costeira da Rocha ${ }^{a}$, João Tomé Saraiva ${ }^{\mathrm{b}, *}$ \\ ${ }^{a}$ ECE Department, Engineering Faculty, University of Porto, Rua Dr. Roberto Frias, 4200-465 Porto, Portugal \\ ${ }^{\mathrm{b}}$ INESC TEC and ECE Department, Engineering Faculty, University of Porto, Campus da FEUP, Rua Dr. Roberto Frias, $4200-465$ Porto, Portugal
}

\section{A R T I C L E I N F O}

\section{Article history:}

Received 25 June 2012

Received in revised form 18 September

2012

Accepted 27 September 2012

\section{Keywords:}

Transmission expansion planning

Long term investments

Discrete evolutionary particle swarm

optimization

Uncertainties

Fuzzy sets

\begin{abstract}
A B S T R A C T
This paper presents a multiyear dynamic model to the Transmission Expansion Planning, TEP, problem to identify the most suitable set of projects as well as their scheduling along the planning horizon. The candidate plans are evaluated using a fitness function that incorporates operation and investment costs plus a set of penalty terms. These terms are associated with the level of losses, non-zero values for the power not supplied namely for the entire system and for $n-1$ contingencies, financial limits, maximum number of projects to implement in each year or all along the horizon and the capability to accommodate not only the expected demand, but also uncertainties affecting the demand forecasts. Given the discrete nature of the problem, we adopted an enhanced approach of the PSO algorithm to solve it. This includes an evolutionary adaptation of the PSO movement rule as well as several modifications to ensure that along the iterative process each candidate solution is technically feasible given its discrete nature. The paper also reports the results of a set of tests to evaluate several design decisions related with the development of the Discrete Evolutionary PSO, DEPSO, as well as to compare the results of its application to the TEP with results reported by other researchers.
\end{abstract}

(c) 2012 Elsevier Ltd. All rights reserved.

\section{Introduction}

In the last 25 years power systems in several countries and geographic areas went through a restructuring process that involved the unbundling of traditional vertically integrated utilities in a number of activities covering the entire value chain from generation to consumption. These activities correspond to generation, transmission and distribution network operation, and retailing together with coordination activities, regarding the technical operation of the system, regulation and whole sale market functions. The unbundling of traditional utilities induced a large number of changes including the introduction of market mechanisms to link the generation and the demand, namely through Market Operators and Bilateral Contracts [1]. In general, this move also implied the development of regulatory mechanisms regarding transmission and distribution network activities (involving the expansion and network reinforcement, the operation and the maintenance) and the correct identification and assignment of all the costs along the value chain. This allows developing tariffs for each activity along the chain and then, using an additive principle, the creation of access tariffs and tariffs paid by final users that adequately reflect the costs each agent brings to the system.

\footnotetext{
* Corresponding author. Tel.: +351 22 2094230; fax: +351 222094150.

E-mail addresses: mz.costeiradarocha@gmail.com (M.C. da Rocha),jsaraiva@ fe.up.pt (J.T. Saraiva).
}

Apart from the changes summarized above, for several years after the beginning of the restructuring process a large emphasis was put on shorter term activities, sometimes neglecting long term planning, in some cases with unpleasant consequences. However, long term studies both at the generation [2] and at the transmission [3] levels continue to be necessary as it is demonstrated by several recent publications, on one side, and by the plans to build a super transmission grid in Europe, on the other. In any case, there has been a change in the models as now generation and transmission agents correspond to different activities and entities. The first one is provided by several competing agents while the second one is typically assigned to a transmission provider or to a Transmission System Operator, TSO, that acts in terms of a regulated natural monopoly, regarding each geographic area. In the past, generation, transmission and distribution were included in the same utility so that long term forecasting and expansion planning activities were developed in a more integrated and centralized way. Now, each generation company develops its own expansion plan taking into account the possible reactions of its competitors and transmission companies should have estimates of the demand evolution and of the possible generation additions in order to prepare expansion and reinforcement plans.

As a result of this unbundling, the TEP problem gained a new complexity degree as the information at the generation and at the demand levels are more uncertain than in the past. In very general terms, a TEP problem aims at determining the timing, the type 
and the location of a set of new transmission facilities that should be added to an existing network along an extended planning horizon in order to ensure an adequate transmission capacity taking into account future generation options and load requirements. Regarding this definition, it is important to make a number of clarifications:

- On the legal and regulatory side, the European Parliament and the European Council approved the Directive 2009/72/CE, establishing common rules for the internal electricity market [4]. TEP is clearly identified as a major responsibility of TSOs, given that they should ensure the long-term ability of the system to meet reasonable demands for the transmission of electricity. On the other hand, the EU Commission Regulation No. $838 / 2010$ of September 23rd 2010, defined the guidelines to the inter-transmission system operator compensation mechanism associated with cross-border electricity flows, to be carried out by the Agency for the Co-operation of Energy Regulators. This ultimately means that transmission providers and TSO's should adequately develop their networks so that they are prepared to accommodate reasonable new generation and demand requirements.

- Secondly, given the complexity of the TEP problem, several researchers developed models that introduced simplifications at two levels as follows:

- In the first place, an extended planning horizon was frequently addressed in a static way, that is, each year in the horizon was treated separated and in sequence, so that the final expansion plan was just a collection of partial plans. This type of approach eliminates the holistic view over the problem given that a particular expansion project justified to address a particular network bottleneck could very well be anticipated in order to address some other network problems given the meshed nature of transmission networks. This means that a multiyear expansion plan is not just the addition of partial plans identified in sequence. The expansion plan should be built using an holistic model that treats the entire planning horizon at a time.

- On the other hand, several researchers simplified the problem using continuous variables to represent the transmission capacity between a pair of nodes. Although computationally more efficient, these continuous versions of TEP will lead to solutions that are not implementable from a technical point of view, given the available conductor sections and voltage levels. This ultimately means that at the end a rounding process was typically used eventually turning the final rounded solution far away from the optimum of the true discrete TEP problem.

- Thirdly and perhaps more than in the past, long term expansion activities are subjected to uncertainties that can now be considered as key elements of any planning process [5]. An expansion plan should be good not only for a reference scenario regarding both the evolution of generation and demand but should also be robust if some changes on this reference evolution happen in the future. This means for instance that the demand can be subjected to uncertainties and that an increasing part of generation is now connected to distribution networks and it is powered by volatile primary resources. In this way, transmission networks should be prepared to accommodate the connection of new generators, uncertain demand as the current economic crisis is showing and the injection of electricity by distribution networks in case the connected distributed generation is larger than the demand of these networks.
As a result of all these concerns, this paper details a multiyear dynamic mathematical model to the TEP problem. The problem is formulated as a single objective discrete optimization problem and it represents all the years or stages in which the planning horizon is discretized. The adopted objective function aims at minimizing the operation plus investment costs along the planning horizon subjected to a number of constraints having technical and financial natures. Given its mixed integer nature, we developed a set of adaptations to the Evolutionary PSO algorithm, in order to turn it more adequate to treat discrete problems. On the other hand, the demand can be subjected to uncertainties modeled by triangular Fuzzy Numbers [6]. This framework is used to evaluate each candidate plan characterizing the risk of eventually not being able to supply the demand subjected to uncertainty. This information is then integrated in the fitness function of the Discrete Evolutionary PSO, DEPSO. According to these ideas, this paper is structured as follows. After this initial section, Section 2 reviews the literature addressing the TEP problem, Section 3 addresses load modeling using Fuzzy Sets, Section 4 details the PSO and the EPSO algorithms and the adaptations in terms of the DEPSO, Section 5 presents the mathematical formulation of the TEP problem and the developed solution algorithm and Section 6 presents results of two Case Studies to illustrate the approach and to compare the obtained results with the ones reported by other researchers for the same networks. Finally, Section 7 presents the most relevant conclusions.

\section{Review of transmission expansion planning models}

Traditionally the expansion planning of transmission networks was addressed in an integrated way with generation expansion planning as detailed in [7]. More recently, integrated generation/ transmission expansion planning approaches continue to be reported in the literature, namely considering geographical areas in which the unbundling of the sector was not implemented $[8,9]$. Under this more traditional and integrated view, it is important to consider that there were approaches that could be used to build expansion plans considering an extended amount of data and forecasts into the future, as well as several software packages designed to evaluate pre-built plans namely computing reliability, transient behavior or stability indices. Typically, these packages were developed by utilities or in research centers closely related with them.

With the advent of the unbundling of the power sector, transmission companies or entities responsible for the preparation of reference transmission expansion plans started to use external information namely related with the construction of new generation stations, the development of distribution networks as demand increases, the increased number of dispersed generation namely connected to distribution networks including recently to LV networks, and the variability of several primary generation resources as wind and solar radiation. In view of these changes, the TEP problem gained new dimensions and it can now be defined as the selection of a set of new transmission equipments (both lines and transformers) and the scheduling of their commissioning along an extended planning horizon. This selection is driven by a criterium or by a set of criteria in case of multi objective approaches $[10,11]$ while enforcing a number of constraints. With the restructuring of power systems, the TEP problem that was already a complex mixed integer optimization problem has to meet new challenges given that:

- More than one entity owns generation assets and the capacity of transmission lines can determine the degree to which generators in different locations can compete. 
- In several countries the developers of wind parks are already asked to pay or at least to contribute to pay new distribution or even transmission connection lines or substation reinforcements.

- There is an increasing substitution effect of transmission by dispersed and volatile generation resources and by demand side management.

- The environmental constraints are now stronger than in the past reducing the number of available corridors and turning more difficult to get licenses to build new high voltage lines.

- Finally, the restructuring of power systems lead to new relations between agents with a progressive decoupling of the flow of electricity from the flow of money requiring the already mentioned creation of specific tariffs to remunerate network providers.

Given all these concerns, the TEP problem typically includes criteria as the maximization of the benefits achieved with the investments (namely reducing power not supplied, losses and operation costs), the minimization of investment and maintenance costs, the reduction of environmental impacts and the increase of the interconnection capacity with other systems. Finally, TSO's often mention the desire to be as neutral as possible in the relations between generation and demand, that is, transmission networks should impose as little as possible constraints to the economic schedules determined by electricity markets. The publications on the TEP problem are numerous as indicated in $[12,13]$, and so it is important to classify the models namely using some general characteristics. In the first place, TEP models can be grouped as:

- Static or single stage models - a TEP model is termed as static or single stage if the planning horizon is just one period. In these cases, the planner aims at identifying the most adequate network for a single future situation. A multiyear horizon is typically decoupled in a number of isolated periods that are treated separately and in sequence. Under this scheme, the planner starts by solving the problem for period 1 and then, when moving to period 2 , the projects scheduled to period 1 are considered as already available. The models detailed in [14-16] are of this type and use optimization techniques as Genetic Algorithms, Tabu Search, Simulated Annealing and Expert Systems.

- Dynamic or multiyear approaches - in this case the optimization problem explicitly includes information regarding the entire planning horizon represented by a number of periods or stages. In these models, the expansion plan is outlined along the entire horizon in a multistage and coordinated way. These approaches are illustrated in [17-19] using traditional optimization techniques, dynamic optimization and a mixed integer formulation.

Regarding the adopted optimization techniques, several techniques and solution algorithms have been applied to the TEP problem. In this scope $[20,21]$ use linear and quadratic programming, [22] uses an interior point approach, [18,23] adopt dynamic programming and $[19,24,25]$ use mix integer formulations. On the other hand, [7,26] adopt Benders decomposition and [27] uses Hierarchical decomposition. More recently, given the complexity and the discrete nature of the TEP problem, metaheuristics also started to be applied. In this scope, Simulated Annealing is used in $[28,29]$, Tabu Search is used in [15,30], and GRASP is adopted in [31]. Evolutionary algorithms as Genetic Algorithms, Evolutionary Programming and Artificial Immune Systems are used in [32-36] and Refs. [35-38] describe applications of Particle Swarm Optimization (PSO) and Ant Colonies (ACO) or provide comparisons of these approaches with other techniques.
Finally, as mentioned in the Introduction, the expansion planning and even the operation of power systems are increasingly influenced by uncertainties. Regarding the TEP, $[5,39,40]$ describe some approaches using scenarios, probabilistic and fuzzy set models.

\section{Load modeling using fuzzy set concepts}

As mentioned above, power systems are affected by uncertainties that should be incorporated in several types of studies and models. In the particular case of TEP studies, if traditional deterministic data is simply used, then the best plans derived from expansion planning exercises will certainly be the most adequate for this crisp input data but may not be robust in the sense that small variations affecting the input data will not eventually be well addressed by such plans. Therefore, incorporating uncertainties, namely in long term models, is an important issue as a way to address the risk of adopting expansion plans too exposed to the uncertainties in input data.

Uncertainties are usually addressed using probabilistic models and more recently using fuzzy concepts that were introduced in the seminal paper of Zadeh [41]. Probabilistic models are well suited for random phenomena in which the event can be reproduced under the same conditions as many times as desired. In this case, there is no uncertainty regarding the possible outcomes of an experiment, but the experiment itself is so complex that it is not actually possible to know in advance which of the possible outcomes will in fact occur. For instance, this is what happens when throwing a die. There is no doubt that the outcome will be face 1 , or face $2, \ldots$, or face 6 , but it isn't actually possible to establish a priori which of these 6 possible outcomes will occur in the next experiment. Differently, fuzzy sets can be used to model phenomena regarding which there is incomplete knowledge or if we want to incorporate data expressed by experts in a qualitative way. For instance, expressions as "approximately" or "larger than" that are common in human language reflect our own subjective past experiences and incorporate knowledge that, in any case, should be included in several models. This issue is of particular importance to model data as, for instance, the evolution of the demand seen by transmission networks. This demand is certainly affected by the current volatile economic environment and by the increasing presence of generation connected to distribution networks, in some cases using volatile primary resources, as wind and solar radiation. In such cases, fuzzy sets represent a very flexible and adequate option to model vague information as for instance associated with expressions as "the demand will be approximately $200 \mathrm{MW}$ ".

The developed TEP model admits that the demand is modeled by fuzzy sets, in particular by triangular fuzzy numbers. A fuzzy set can be defined by (1) as a set of ordered pairs in which $x_{1}$ is an element of the universe $X$ under analysis and $\mu_{\tilde{A}}\left(x_{1}\right)$ is the membership degree of $x_{1}$ to the fuzzy set taking values in $[0.0 ; 1.0]$ and it expresses the compatibility degree of $x_{1}$ with the fuzzy set.

$\tilde{A}=\left\{\left(x_{1} ; \mu_{\tilde{A}}\left(x_{1}\right)\right), \quad x_{1} \in X\right\}$.

The membership function of triangular fuzzy numbers have a particular shape as it is illustrated in Fig. 1, and can be used to model the knowledge associated with an expression as "the demand will be approximately $200 \mathrm{MW}$ ". Taking the number in Fig. 1, the most credible value that the demand can take is

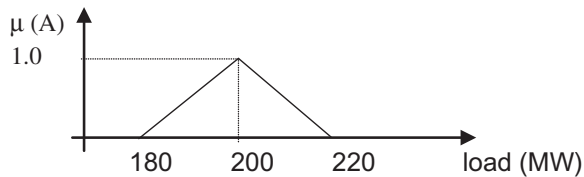

Fig. 1. Illustration of a triangular fuzzy number 
200 MW but the planner wouldn't like to completely discard values from 180 to $200 \mathrm{MW}$ on one side and from 200 to $220 \mathrm{MW}$ on the other. According to his assessment, this means that values in the intervals [180;200[ and ]200; 220] MW are still possible instances of this fuzzy set. Given its particular shape, a triangular fuzzy number as the one in Fig. 1 is denoted by $(180 ; 200 ; 220)$. Regarding a fuzzy number, an $\alpha$-level set or an $\alpha$-cut corresponds to the crisp set of values having a membership degree larger than or equal to $\alpha$ and the central value is the mean value of the 1.0-cut. In the case of the number in Fig. 1, the 0.0-cut is the interval $[180 ; 220]$ and the central value is 200.0.

Finally, if a multiyear TEP problem is addressed, the planner also has to specify a load growth coefficient, $\lg r$ in percentage, that is used to transfer the load in period $p d$ of the planning exercise to the period $p d+1$. Using this coefficient, if the demand in node $k$ in period $p d$ is given by the triangular fuzzy number $(180 ; 200 ; 220)$, then in period $p d+1$ it will be given by the triangular fuzzy number $\left(\frac{100+\lg r}{100} .180 ; \frac{100+\lg r}{100} \cdot 200 ; \frac{100+\lg r}{100} \cdot 220\right)$.

\section{Discrete evolutionary particle swarm optimization, DEPSO}

\subsection{PSO}

Particle Swarm Optimization (PSO) was originally proposed in 1995 by Kennedy and Eberhart [42], and is based on the analogy of swarms of birds and fish schooling. PSO is based on the idea of each agent being a particle, characterized by its position and velocity. A set of particles forms a population or swarm and each particle evolves according with the so called movement rule. The ultimate objective of the particles consists in finding the position which corresponds to the best possible performance in the search space. A new particle is generated from an ancestor, according to the "movement rule" and this scheme proved to be adequate to make the swarm converge to the zone of the optimum, but failed to assure the convergence to an accurate optimum final position.

\subsection{EPSO}

Evolutionary Particle Swarm Optimization (EPSO) is an evolutionary meta-heuristic proposed in 2002 by Miranda and Fonseca [43]. The EPSO particle structure and behavior is similar with that of PSO. Each solution is coded in a particle, in its original or phenotypic variables. The EPSO algorithm structure is illustrated below.

\footnotetext{
Procedure EPSO

Initialize a random population $P$ of $n p t$ particles REPEAT

Replication - each particle is replicated $r$ times;

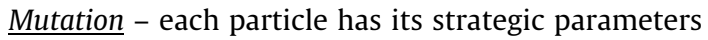
mutated;

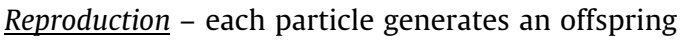
through recombination;

Evaluation - each offspring has its fitness evaluated;

Selection -the best particles survive to form a new generation;

Test - for termination (based on fitness, on number of generations or other criteria);

Until test is positive.

End EPSO
}

Adopting the PSO movement rule to recombine the particles together with self-adaptation schemes, EPSO improves the convergence speed and develops a sense of self-adaptation, reducing the dependence on a number of pre-set weights. In fact, EPSO may be seen either as an evolutionary computing method of the self-adaptive (SA) evolutionary strategy type, with a special rule for the replication of individuals instead of ordinary crossover and mutation of object parameters, or as a special PSO method where the weights that condition the movement in space undergo self-adaptive mutation. In classical approaches to EPSO, each particle is cloned twice so that one can perform the search using two populations. The mutation of the strategic parameters $W$ into $W^{*}$ is done using either (2), (3) [43]. In these expressions, $\tau$ and $\sigma$ are learning parameters fixed externally.

Multiplicative Lognormal random numbers

$W_{p t}^{*}=W_{p t} \cdot[\log N(0,1)]^{\tau}$

Additive Gaussian distributed random numbers

$W_{p t}^{*}=W_{p t}+\sigma \cdot N(0,1)$

In the most effective EPSO variant, not only the weights affecting the components of the movement are mutated but also the global best $\left(b_{G}\right)$ particle is randomly disturbed, according to (4) [43].

$b_{G}^{*}=b_{G}+W_{p t 4}^{*} \cdot N(0,1)$

The weight $W_{p t 4}$ controls the "size" of the neighborhood of $b_{G}$ where it is more likely to find the real best solution. The off-springs are generated by recombination of the particles. This recombination defining the movement rule is given by (5) and (6), and it is illustrated in Fig. 2.

$X_{p t}^{i t+1}=X_{p t}^{i t}+V_{p t}^{i t+1}$

$V_{p t}^{i t+1}=W_{p t 1}^{*} \cdot V_{p t}^{i t}+W_{p t 2}^{*} \cdot\left(b_{p t}-X_{p t}^{i t}\right)+W_{p t 3}^{*} \cdot\left(b_{G}^{*}-X_{p t}^{i t}\right) \cdot p$

In these expressions:

- $X_{p t}^{i t}$ is the location of the particle $p t$ in generation $i t$.

$-b_{p t}$ is the best point found by particle $p t$ in its past life up to the current generation.

- $b_{G}^{*}$ is the best overall point found by the swarm of particles in their past life.

- $V_{p t}^{i t}$ is the velocity of particle $p t$ at generation it.

- $W_{p t 1}^{*}, W_{p t 2}^{*}, W_{p t 3}^{*}$ and $W_{p t 4}^{*}$ are the mutated weights conditioning the inertia, the memory, the cooperation terms and the best global particle.

$-p$ is a communication factor in $[0,1]$. The vector associated with the cooperation factor does not point exactly to the global optimum $b_{G}$ but to a mutated nearby location as described in [44].

The first step on the selection process is the evaluation of all the offsprings. This evaluation step is conducted using a fitness function that can include penalty terms to enforce constraints. The best fitness may correspond either to the largest or to the minimum value of the fitness function, depending on the nature of the problem

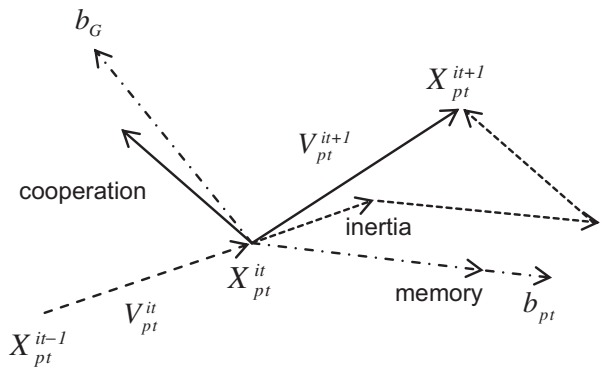

Fig. 2. Illustration of the EPSO movement rule. (Source: [44]) 
being addressed. The offspring selection may be done by stochastic tournament or other selection procedures.

When comparing EPSO with classic PSO, Ref. [44] reports that EPSO displays faster convergence, it is more accurate and more robust and it is more insensitive to weight initialization.

\subsection{Discrete PSO, Chaos and Lamarkism}

The original PSO model was developed to tackle problems in continuous search spaces. However, many real problems have discrete nature and recently different discrete PSO models were proposed. The first discrete PSO approach was proposed by Kennedy and Eberhart for binary-valued solution elements [45]. The entire algorithm of the binary version of PSO is almost the same as that of the basic continuous version, except for the state equations listed below. The position of each particle is a vector in the d-dimensional binary solution space, $x_{i} \in\{0,1\}^{d}$ and the velocity is a vector in the $d$ dimensional continuous space. In [45], the probability of an agent's deciding yes or no, true or false, or making some other decision is a function of personal and social factors as suggested by Eq. (7). The parameter $V_{p t, j}^{i t}$ is an agent's alternative choice tendency and determines a probability threshold in the range $[0,1]$. If $V_{p t . j}^{i t}$ is higher, the agent is more likely to choose 1 , and lower values favor 0 choice. One of the functions accomplishing this threshold range is the sigmoid function (8), which is usual in artificial neural networks.

$P\left(X_{p t, j}^{i t+1}=1\right)=f\left(X_{p t, j}^{i t}, V_{p t, j}^{i t}\right.$, pbest $_{p t, j}$, gbest $\left._{p t, j}\right)$

$\operatorname{sig}\left(V_{p t, j}^{i t}\right)=\frac{1}{1+\exp \left(-V_{p t, j}^{i t}\right)}$

Like in the basic PSO continuous real version, the formula for the discrete binary PSO version can be described by

$V_{p t, j}^{i t+1}=V_{p t, j}^{i t}+\operatorname{rand}_{1} \cdot\left(\right.$ pbest $\left._{p t, j}-X_{p t, j}^{i t}\right)+\operatorname{rand}_{2} \cdot\left(\right.$ gbest $\left._{j}-X_{p t, j}^{i t}\right)$

If $\rho_{p t, j}^{i t+1}<\operatorname{sig}\left(V_{p t, j}^{i t}\right)$, then $X_{p t, j}^{i t+1}=1$ else $X_{p t, j}^{i t+1}=0$

In these expressions rand $_{1}$ and rand $_{2}$ are positive random numbers, drawn from a uniform distribution, with a predefined upper limit; $\rho_{p t, j}^{i t+1}$ is a random number between 0 and 1 . These equations are used in an iterative repeated way over each $j$ dimension of each particle. The second and the third terms in (9) can be weighted like in the basic continuous version of PSO. $\operatorname{sig}\left(V_{p t, j}^{i t}\right)$ should not get too close to the limits of the interval $[0,1]$ to ensure good chances for bit flipping. This can be achieved by limiting the maximum value of $V_{p t . j}^{i t}$, which is often set in $[-4,+4]$.

Discrete PSO approaches also differ from traditional PSO regarding encoding. The particles are vectors composed of integers, instead of real numbers, and several researchers studied different codification alternatives as, for instance, Gallego et al. [46] and Jin et al. [47]. Binary representations are the most common, although [46] mentions several reasons for not using them in the TEP problems, namely that mutation and cross-over can generate off-springs too different from their parent configurations eventually originating chaotic behavior. Jin et al. [47] formulated the discrete velocity vector of the PSO algorithm using (11). In this expression Fix is a function that takes the integer part of its argument and $t_{\text {int }}$ tin, $t_{\text {mem }}$ and $t_{\text {coop }}$ are the usual inertia, memory and cooperation terms of the PSO velocity vector.

$v_{p t}^{i t+1}=F i x\left(t_{i n t, p t}^{i t}+t_{m e m, p t}^{i t}+t_{c o o p, p t}^{i t}\right)$

Excess attraction promoted by the best particles leads to premature convergence of the algorithm to solutions that can be far from the best. The literature presented different ways of approaching this problem, and one of the most interesting and promising includes the concept of chaos. Caponetto et al. [48] showed that chaotic sequences improve the performance of Evolutionary Algorithms. In addition to the ability of enlarging the exploration of the search space, chaos-based approaches also allow for faster convergence, contributing to improve the performance of the algorithms. In this scope, Malik et al. [49] proposed a PSO optimizer using a sigmoid function to determine the weights. Several experiments showed that the performance improved with faster convergence capability and aggressive movement towards the solution region. Finally, Houck et al. [50] investigated the benefits of including a Lamarkian flavor in the evolution strategy. As detailed in Section 4.4, when addressing discrete problems it is frequent that the velocity vector gets zero meaning that the particle would remain unchanged. If that is the case, one can promote a change of some dimensions of the particle meaning that one would act at the fenotype level, rather than at the components used to determine the velocity vector, that is, instead of changing the particle at the genotype level. This ultimately means that one is promoting a local search around a particle that will eventually already have promising characteristics.

\subsection{DEPSO}

The discrete EPSO used in this work is a new approach of the EPSO model able to tackle problems with non-continuous and integer search spaces and it was originally described in [51]. Even though it includes the same main blocks of classic EPSO, DEPSO has the following main differences when compared with classical EPSO: the elements in the particles are integers, the mutation rules are based on chaotic behaviors and it is introduced a third population with Lamarckian evolution.

A solution for a job scheduling problem, also named as particle, is represented by a vector whose positions represent the state when each project will be available for use. In addition to these states, two more are possible, corresponding to situations of nonimplementation of a particular project: either the possibility of not being chosen, or the possibility of being postponed beyond the periods under review. Admitting that npd is the number of periods in the planning horizon, this means that $n p d+2$ possible states are possible. Accordingly, each population is a set of particles and is characterized by the number of particles $n p t, p t \in\{1 \ldots n p t\}$, by the number of projects $n p j, p j \in\{1 \ldots n p j\}$ and by the number of possible periods in the horizon, $p d \in\{0 \ldots n p d+1\}$.

The off-springs are generated by recombination of the particles, following recombination rules similar with EPSO. As in the classical EPSO approach described in $[43,44]$, the inertia, memory and cooperation weights are subjected to mutation. Regarding the classical EPSO, a different mutation operator was adopted (12). For each iteration $i$, particle $p t$ and period $p d$ it includes a sigmoid function with chaotic behavior. In (12) as well as in expressions used along the paper the symbol $*$ denotes a value or parameter that underwent mutation.

$w_{p t, p j, p d}^{i t+1^{*}}=\left(0.5+\operatorname{rand}()-\frac{1}{1+\exp \left(-w_{p t, p j, p d}^{i t^{*}}\right)}\right)$

This mutation rule includes the previous value of the weight to mutate, that is, it carries along the influence of the weights of the previous iterations to the following ones. It also allows an easy integration in the classical mutation expressions, contributing to design a simpler and faster algorithm. The best global particle is a vector whose positions are mutated only when randomly generated numbers take values less than $k_{c} \in[0,1]$.

The mutated $b_{G}$ is given by (13) in which the weights $w_{4}^{i t}$ also undergo mutation using (12). 
$b_{G p j}^{*}=b_{G p j}+\operatorname{round}\left(2 \cdot w_{4}^{i t^{*}}-1\right)$

A major difficulty of using particle swarms is the control of the population movement, ruled by velocity $V$. In the case of DEPSO this situation is even more relevant, since the space is not continuous, and one must ensure that the movement rule makes the particles move from one position to another while respecting the search space.

$X_{p t, p j}^{i t+1}=X_{p t, p j}^{i t}+V_{p t, p j}^{i t+1}$

$V_{p t, p j}^{i t+1}=\operatorname{round}\left(w_{p t, p j, 1}^{i t^{*}} \cdot V_{p t, p j}^{i t}+w_{p t, p j, 2}^{i t^{*}} \cdot\left(b_{p t, p j}-X_{p t, p j}^{i t}\right)+w_{p t, p j, 3}^{i t^{*}} \cdot\left(b_{G, p j}^{*}-X_{p t, p j}^{i t}\right) \cdot P_{p j}\right)$

The movement rules in DEPSO are similar to those of EPSO, but all elements in $X$ and $V$ in (14) and (15) are integers [51]. It should be noted that expression (15) includes a rounding process to obtain integer elements in $V$. When the particles exceed the search space boundaries, they are repaired typically returning them back to the search space by placing them on the nearest integer position in the search space.

In the developed algorithm we also adopted a third population to promote diversity or local search, according to the progress of the algorithm. When the velocity of a particle is zero for the particles in populations 1 and 2 and for all particles of population 3 , a Lamarckian evolution is promoted to define $V$. This evolution mirrors the ideas of Jean Baptiste Lamarke, a French biologist that lived in the end of the XVIII century and that can be seen as a proto evolutionist. According to his ideas described for instance in [50], living beings would suffer mutations along time at the macroscopic level in order to generate more complex and perfect entities progressively more adapted to their living conditions. Later on, the evolution theory of Darwin privileged mutations at the genotype or microscopic level, rather than at the fenotype or macroscopic level, suggested by Lamarke. In the developed DEPSO approach, if as a result of genotype changes on the three terms of the velocity vector, this vector is null, then it is promoted a Lamarkian based evolution admitting that some elements of the velocity vector are changed. These changes are considered if randomly generated numbers $N(0,1)$ are smaller than a threshold $k_{\text {Lam }} \in[0,1]$.

The general structure of the DEPSO algorithm is detailed below. The application of DEPSO to the TEP problem will be detailed in Section 5.3.

\section{Procedure DEPSO}

Initialize a random population $P$ of $n p t$ particles; REPEAT

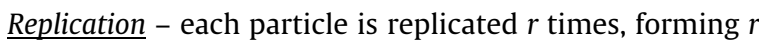
populations;

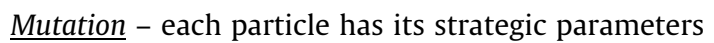
mutated using expression (12) and the best global particle is mutated using (13);

Reproduction - each particle generates an offspring through recombination using expression (14) and (15). If the offsprings exceed the search space boundaries they are returned back to the search space using a repair strategy. If the velocity vector (15) of an offspring is zero, then a Lamarkian based evolution step is activated;

Evaluation - all offsprings in all $r$ populations are evaluated using a specified fitness function;

Selection - particles $p t$ from population 1, population 2 , ..., population $r$ are compared using the respective values of the fitness function. Regarding particle $p t$, it survives the particle in population $r$ that has the smallest value of the fitness function, in case of minimization problems. At the end of this selection step, a new population having $n p t$ particles is formed;

$\underline{\text { Test }}$ - for termination (based on fitness, on number of generations or other criteria);

Until test is positive.

End DEPSO

Finally, it is important to mention that the DEPSO model can accommodate constraints by using additive penalties in the evaluation function. The merits of DEPSO result from the EPSO own merits. In addition, as DEPSO was specially designed to address integer problems, when treating such problems it shows some advantages: it displays a faster convergence, it is more accurate and it is more robust. DEPSO does not require adjustment in the parameterizations, it shows an easy convergence, it allows smaller populations (which reduces the number of evaluations and reduces the time required for each run) and it is able to escape from local minima.

\section{Mathematical formulation of the TEP problem}

\subsection{Introduction}

A dynamic or multiyear TEP problem aims at determining the timing, the type and the location of the new transmission facilities to be added to an existing network along an extended planning horizon in order to ensure an adequate transmission capacity taking into account future generation options and load requirements. This problem is typically addressed along a long-term horizon, organized on a yearly basis and with all sub periods considered jointly in order to model the influence of investments in a given period over the network performance in other periods. TEP models are usually very complex, given their mixed integer nature and the adopted solution algorithms, leading to a significant computational burden.

The initial network should be characterized with information about power lines and underground cables, substations and transformers. For planning purposes, lines and transformers can be treated as branches of the network, provided that one keeps in mind the technical and economic characteristics that differentiate them.

The characterization of each generation center includes the type of station, location, identification of the connection node, number of generation units and characteristics of each unit. In order to guarantee the feasibility of the model, and without prejudice to the results to be obtained, we will consider linear generation cost functions in order to evaluate operation costs. Economic data, such as inflation, interest and discount rates for the long term are set based on forecasts that, as for other factors mentioned above, may be subject to uncertainties.

\subsection{The definition of the expansion plan}

An expansion plan is a set of infrastructure network investments, scheduled over the planning horizon. Investments have to be selected according to various criteria, and be considered as a whole in a coordinated manner. There is an important dynamic component to be considered, since investments in a given year may have impact in the following years in different areas, some of them geographically distant. So, in response to operating conditions for the years included in the planning horizon, alternative expansion plans can be prepared and should be evaluated according to the selected criterium. After appropriate evaluation of the different plans, decisions are made on the investments, based on the plans that were considered more appropriate. 
The problem is so complex that assumptions have to be done in order to make the TEP exercise feasible:

- Basic assumptions: load growth rates; expansion by adding new lines or transformers with characteristics similar to the ones in the same corridor; the reliability focus is restricted to transmission lines or transformers; only load uncertainties modeled by triangular fuzzy numbers are assumed.

- List of projects: new lines or transformers can be installed not only in existing corridors or substations but also connecting new demand or generation centers or being established in new corridors. In any case, the cost of each project is admitted to be known and it is admitted that there is no precedence and no relationship among the projects. Apart from their cost and nodes, as a result of the TEP exercise each project will be associated to the period in which it will enter in service.

The TEP project list is based on npj projects, and a time frame based on $n p d+2$ periods, typically associated to yearly stages. Dynamic TEP problems usually have temporal horizons that can go from 1 year to several years, rarely exceeding 10 years. Briefly, the definition of the expansion plan project list includes the following topics: number of states or years, list of projects available for implementation admitting that they have already been cleared on environmental evaluations, available financial resources and a minimum threshold for a quality of service index. A solution $X_{p t}$ of the TEP problem will then be associated to a particle in the population of the DEPSO algorithm and it corresponds to a plan that includes a number of projects selected among this list, including their time frame. The search space under analysis is discrete and integer and it typically includes a large number of possible alternative plans, given by $(n p d+2)^{n p j}$.

\subsection{Mathematical model}

Each particle is a possible solution to the problem and the main difference between this approach and classical EPSO is that the elements in this particle are integers, and not real. The integrity of the particle is maintained all along the solution algorithm as mentioned in Section 4.4. In the DEPSO, a population is characterized by:

- The number of particles, npt, representing the population size and associated with the index $p t$. In the developed approach we used fixed sized populations along each run of the algorithm.

- The number of positions in each particle, $n p j$, representing the particle size and associated to the index $p j$.

- And the number of periods of the planning horizon, npd, associated with the index $p d$.

A population can then be seen as a matrix having $n p t$ lines and $n p j$ columns. This means that a column in this matrix corresponds to a particle in the population that has npj positions, each one associated to a project in the list of possible projects to be supplied by the planner. Each project can eventually be selected to incorporate this particular particle, that is, to be included in a particular plan. Each position of this particle, that is, each position of this column of the matrix, is filled with an integer indicating the period in the horizon regarding which this particular project is scheduled. As indicated in Section 5.2, although the planning horizon has npd periods, we consider two extra values, the 0 indicating that the project was not selected to integrate this particle and $n p d+1$ indicating that it was postponed.

Having in mind these considerations, the formulation of the TEP model can thus be given by (16)-(21). This represents a condensed and general formulation that in fact is not different from the formulations available in several publications listed in Section 2. In general, the TEP problem aims at identifying the most adequate expansion plan by selecting particular projects from a project list and by adequately locating them along the planning horizon, so that a cost function is minimized while enforcing a number of constraints. Different publications report particular variations over this general formulation in the sense that, for instance, financial or reliability constraints are modeled in different ways. The particular characteristics of the implemented TEP model will be detailed below regarding both the terms in the objective function and the formulation of each set of constraints. Finally, it should be noticed that this general formulation has a mixed integer nature due to the fact that the planner provides a list of possible expansion projects, from which some of them are used to build the expansion plan. On the other hand, using the DEPSO algorithm, in each iteration there are a number of possible plans in the population (corresponding to the particles). Therefore, each solution $X$ is indexed to the indices $p t$ associated to the particle under analysis and it related to the iteration of the algorithm.

$\min \operatorname{Cos} t X_{p t}^{i t}=\sum_{p d=0}^{n p d+1}\left[\sum_{p j=1}^{n p j} I C_{p j} \cdot K_{p t, p j}^{p d}+O C_{p t, p d}\right] /(1+i r)^{p d}$

Subjected to:

Physical constraints;

Financial constraints;

Reliability constraints;

$p t=1,2, \ldots, n p t$

it $=1,2, \ldots$, niter

This problem aims at minimizing the objective function (16) that includes operation and investment costs along the horizon referred to the initial year using a discount rate $i r$, assuming that the planning horizon is multiyear. Investment costs, $I C_{p j}$ result from the sum of the updated values for each project $p j$ included in the solution under analysis, that is, associated with particle $p t$, considering the interest rate defined in the beginning of the process. In (16) $K_{p t, p j}^{p d}$ represents a binary variable that in case of being 1 indicates that project $p j$ in the project list is included in particle $p t$ and scheduled to start operation in period $p d$. Operating costs are given by $O C_{p t, p d}$ and are associated to the costs in period $p d$ related with the particle $p t$. These costs can include the generation costs, losses, maintenance and costs associated with ancillary services, and externalities. As it is necessary to use simple models, usually linear optimization models are adopted to estimate these operation costs.

The feasibility of solutions $X_{p t}^{i t}$ and their operational cost, in particular Power Not Supplied, PNS, are evaluated running a DC Optimal Power Flow for each period pd in the horizon. In each period the network integrates the selected installations completed previously and the forecasted demand.

The model (16)-(21) considers three types of constraints that are included in the fitness function of the DEPSO algorithm using penalty terms. These constraints and the respective penalty terms will now be detailed:

- Physical constraints - the formulation includes a number of constraints as physical limits of the network branches, physical limits of the generators, number of projects that can be developed simultaneously, value of a reliability index to ensure the quality of the expansion plan. Several of these constraints are inherently related with power flow algorithm. The power flows 
in each period are calculated by solving a deterministic DC OPF exercise formulated by (22)-(26). In this formulation $c_{k}, P g_{k}$ and $\mathrm{Pl}_{k}$ represent the variable generation cost, the generation and the load connected to node $k, G$ is a penalty assigned to Power Not Supplied, PNS, $a_{b k}$ is the sensitivity coefficient of the active flow in branch $b$ regarding the injected power in node $k, \mathrm{Pg}_{k}^{\mathrm{min}}$ and $P_{k}^{\max }$ are the minimum and maximum outputs of the generator connected to node $k$, and finally $P_{b}^{\min }$ and $P_{b}^{\max }$ represent the minimum and maximum active power flow in branch $b$.

$\min f=\sum \mathrm{c}_{\mathrm{k}} \cdot P g_{k}+G \cdot \sum P N S_{k}$

Subject to:

$\sum P g_{k}+\sum P N S_{k}=\sum P l_{k}$

$P g_{k}^{\min } \leqslant P g_{k} \leqslant P g_{k}^{\max }$

$P N S_{k} \leqslant P l_{k}$

$P_{b}^{\min } \leqslant \sum a_{b k} \cdot\left(P g_{k}+P N S_{k}-P l_{k}\right) \leqslant P_{b}^{\max }$

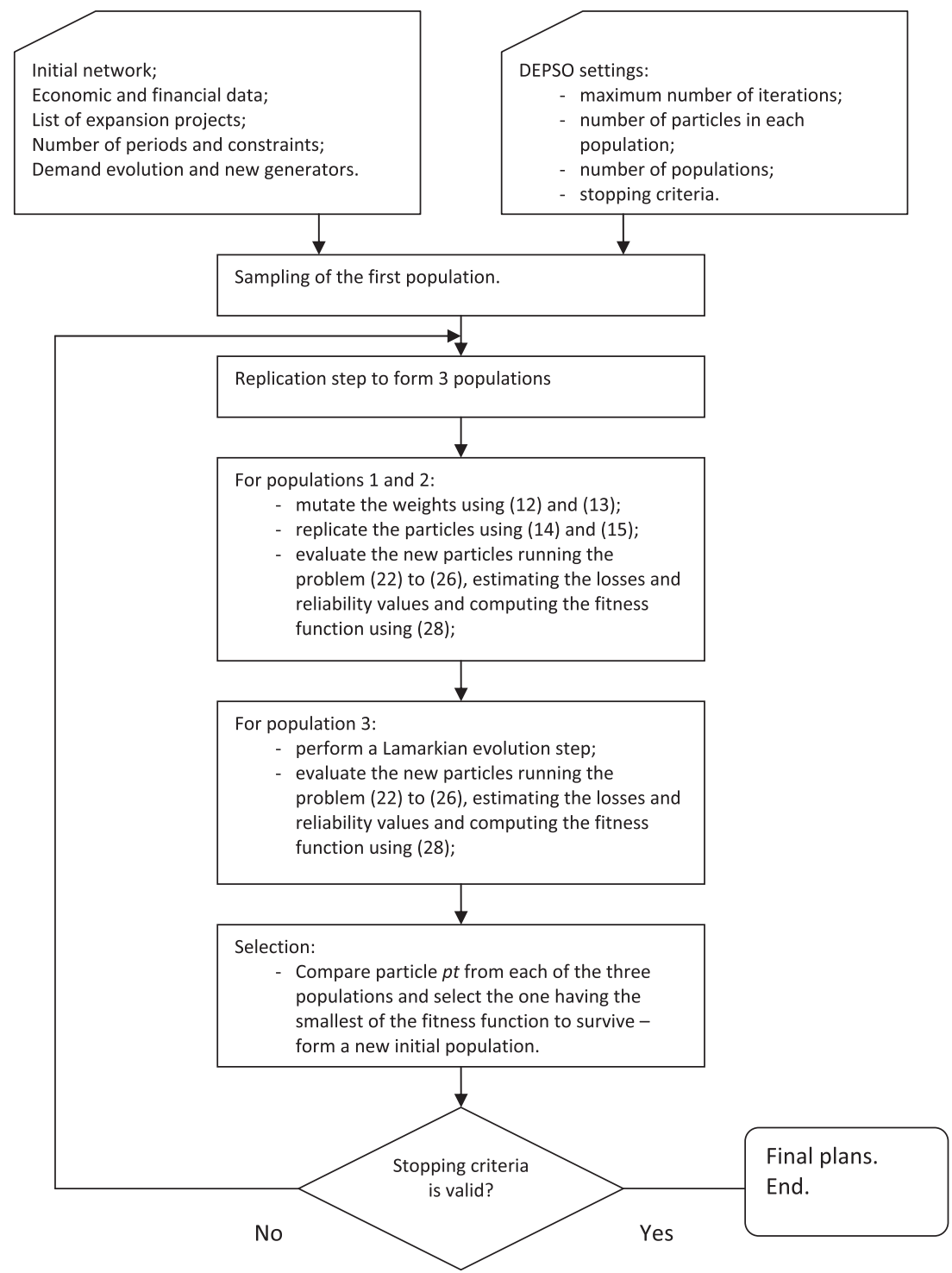

Fig. 3. Flowchart detailing the main blocks of the developed TEP algorithm.
In order to increase the realism of the model, this DC-OPF was enhanced to include an estimate of transmission losses. Considering that voltage magnitudes are $1.0 \mathrm{pu}$, active losses in branch $\mathrm{b}$, from node $i$ to node $j$, are approximately calculated by (27). In this expression $g_{i j}$ is the conductance of branch $i-j$ and $\theta_{i j}$ is the phase difference across this branch.

$\operatorname{Loss}_{i j} \approx 2 . g_{i j} \cdot\left(1-\cos \theta_{i j}\right)$

At the end of each DC-OPF run, voltage phases and branch losses are computed, and half of the losses in each branch are added to the load in each extreme bus. This change on loads requires adjusting the generation level and thus the voltage phases. These calculations are repeated until convergence is reached. The level of losses estimated at the end of this process is then compared with a reference value. If this reference value is exceeded, then a penalty pen $n_{1}$ is included in the fitness function. In a similar way, the solution of the DC-OPF algorithm (22)-(26) also outputs the value of PNS. If the value of PNS admitting that all systems components are available, $\operatorname{PNS}(n)$, is not zero, then the penalty term pen $_{2}$ is also added to the fitness function of the particle under analysis.

- Financial constraints - two financial constraints are considered in the model. The first one corresponds to the maximum 
number of projects that can be implemented per period. This limitation arises due to financial or operational reasons and, if it is violated, we consider a penalty term $p^{2} n_{3}$ in the fitness function. The second one corresponds to the maximum investment value over the entire horizon and it models a global financial constraint. If the investment level associated with the projects scheduled in particle $p t$ exceeds the maximum admitted value, then a penalty pen $_{4}$ is included in the fitness function.

- Reliability constraints - the developed approach penalizes plans in which PNS is non-zero for configurations of the network associated to $n-1$ contingencies. In some countries, the TSO operation code also includes a list of $n-2$ contingencies regarding which the system should survive. If that is the case, the contingencies in this list should also be tested for the system configuration associated with particle $p t$ and, if non-zero PNS values are obtained, then a penalty term pen $_{5}$ is also used.

Considering the objective function (16) plus the five penalty terms mentioned above, we formulate the fitness function of the TEP problem by (28).

$\min \operatorname{Cost} X_{p t}^{i t}=\sum_{p d=0}^{n p d+1}\left[\sum_{p j=1}^{n p j} I C_{p j} \cdot K_{p t, p j}^{p d}+O C_{p t, p d}\right] /(1+i r)^{p d}+\sum_{t=1}^{5} p e n_{t}$

Regarding this expression, it should be noticed that each particle corresponds to a possible solution to the TEP problem, that is, to a possible expansion plan. Each particle will then have to be characterized in terms of its goodness regarding a fitness function. If all constraints were enforced by the plan associated to a given particle, then its fitness function would only correspond to the cost function (16). However, it is likely that the plans associated with several particles violate some constraints and so the corresponding particles will not be as good as others. This information is included in the fitness function by adding penalty terms to the cost function (16) thus arriving to (28). These penalty terms are assigned large positive values so that a particle leading to the violation of several constraints will display a large value of the fitness function. Given that the problem is a minimization one, these largely penalized solutions tend to be eliminated from the population as the iterative process develops.

It is also important to notice that in this approach each penalty term is activated as soon as the associated constraints are violated. This corresponds to a conservative approach in which the planner admits no violation of any constraint in the final expansion plan. As a result, the value of each penalty term is not dependent on the amount of violation of each constraint limit. Therefore, the value of the penalty terms can be set a priori and have the same units as the rest of the terms in the cost function. In the simulations to be reported in Section 6 all the penalty terms were assigned a value of 100,000\$ and each of these values is added to the fitness function if each of the corresponding constraints are violated. It is clear that a different approach could have been adopted, for instance considering penalty terms including a factor that multiplies the amount of violation of each constraint limit. If that was the case, each of these factors would then have different units, depending on the nature of each constraint.

Due to the evaluation process complexity, in our case based on running a large number of DC OPF problems, a simple, but effective analysis of the particles is adopted so that only those particles with suitable characteristics are fully evaluated. The evaluation process includes three distinct phases: the evaluation of the eligibility of the particles $X_{p t}^{i t}$; the evaluation of solutions obtained for the deterministic demand level; and the assessment of the best population, admitting that the demand is affected by uncertainties modeled by triangular fuzzy numbers.

The first steps of the multiyear TEP algorithm admitting a deterministic demand level are the initial data definition and the DEPSO settings. Complementary, a selected particle may be used for seeding purposes, which may accelerate the convergence. The first population is generated with so many particles as those defined in the initial data. The multiyear TEP model with deterministic demand is then structured as indicated below and Fig. 3 details the main blocks of the developed approach.

Procedure TEP with deterministic demand

Initialize a random population $P$ of $n p t$ particles;

Repeat

Replication - clone the population 3 times;

Populations 1 and 2

Mutation - each particle has its strategic parameters mutated;

Reproduction - each particle generates an offspring through recombination;

Evaluation - each offspring has its fitness evaluated; Population 3

Mutation/Reproduction - each particle is mutated and generates an offspring by Lamarkian evolution;

Evaluation - each offspring has its fitness evaluated;

Selection - the best particles survive to form a new generation;

$\underline{\text { Test }}$ - for termination, based on fitness and on number of generations;

Until test is positive.

End TEP with deterministic demand

\subsection{Treatment of demand uncertainties and solution algorithm}

Load data may be subject to uncertainties. These uncertainties may be associated with the demand level in each period and at every bus bar. In the developed model, we admitted that loads are represented by triangular fuzzy numbers, as detailed in Section 3 . Using these load representations we are implicitly admitting that there is an infinite number of possible load combinations against which the robustness of each plan should be tested.

In order to simplify and to speed up this evaluation process, we considered that the triangular fuzzy numbers representing each of the loads are discretized in a number of $\alpha$-cuts defined in Section 3. In particular, in the developed approach we considered $\alpha=1$, $\alpha=0.5$ and $\alpha=0$.

In the first case, $\alpha=1$ corresponds to a single load combination in which all nodal load values have a membership function of 1 . In fact, the expansion plans associated to each of the particles in the final population of the DEPSO algorithm are associated with this single load combination. This means that the solutions identified so far can be termed as deterministic solutions, or expansion plans associated with a set of load values in the absence of uncertainties.

For $\alpha=0.5$ and $\alpha=0$ each load is represented by an interval corresponding to the load values having a membership degree not smaller than 0.5 and not smaller than 0.0. Each particle in the final population is then tested using the load combinations in these $\alpha$-cuts. In particular, we are interested in checking if PNS assumes non zero values if the load in each node is allowed to vary in the intervals associated with these cuts. After extensive tests we concluded that an accurate indication on the PNS value associated with a particle within a specific $\alpha$-cut could be provided just considering the combination of the largest possible values that each nodal load could assume in the corresponding $\alpha$-cut. This means using for each load the largest value in the interval defining the cut under analysis. If, from this analysis, there is a non-zero 
outcome for PNS associated with a particle $p t$ in the final population, then the fitness function (28) includes a new penalty term as indicated in (29).

$\min \operatorname{Cost} X_{p t}^{i t}=\sum_{p d=0}^{n p d+1}\left[\sum_{p j=1}^{n p j} I C_{p j} \cdot K_{p t, p j}^{p d}+O C_{p t, p d}\right] /(1+i r)^{p d}+\sum_{t=1}^{6} p e n_{t}$

Once all the particles in the final population are analyzed considering the specified load uncertainties, we get the final value of the fitness function for all particles. Using these values, we can finally select the most adequate transmission expansion plan.

\section{Case studies using the DEPSO algorithm}

\subsection{General aspects}

The tests of the multiyear TEP model using DEPSO were carried out using two networks:

- The Garver Network, introduced by Garver in 1970 [52].

- The IEEE RTS Network, with 24 busbars. The demand is set at 8.550 MW and the installed generation capacity is set at 10.215 MW, three times more than the original values specified in [53].

These networks were chosen for the following reasons: they are reference networks in many scientific TEP studies and the result of the static planning is known in both cases. In addition, we wanted to test the dynamic TEP with DEPSO with a network that would be larger than the ones usual in the literature on dynamic TEP, as for example in $[35,54]$.

The first tests, using the Garver Network, were conducted to gain insight on the behavior of the DEPSO algorithm, namely regarding its settings. The tests are normally performed in sets of 10 or 100 trials for each setting option, to assess the frequency of occurrence of the expected result. The following topics were investigated: population dimension; seeding procedure; diversity in the population; chaotic mutation operator; mutation of the best global; communication factor $p$; the Lamarckian evolution when $V=0$, in populations 1 and 2 and the Lamarckian evolution, in population 3 (namely the most adequate value for the threshold $k_{\text {Lam }}$ mentioned in Section 4.4). As a result of these runs, it was possible to parameterize the DEPSO algorithm considering a number of key aspects that are listed in Table 1. Apart from these key DEPSO parameters, this table also includes some other parameters that were used when running the TEP exercise using the Garver network and the IEEE RTS system. It is clear that some of these values have to be changed if another network is analyzed. The number of projects in the list to be specified by the planner is just an example of such a situation.

Finally, the next paragraphs list the input data and the results to be obtained from a TEP exercise:

- Input data - it can be gathered in three main groups:

- Network data - network in the period before starting the planning exercise (topology of the network, branches, extreme buses and resistance and reactance, nodal loads and generators including installed capacity and connection buses), load growth coefficient, definition of the triangular fuzzy numbers to model load uncertainties, generators to be connected to the network along the planning horizon.

- Planning data - this includes the number of periods in the horizon, the list of projects (for each project this includes the extreme buses, the transmission capacity and the investment cost), maximum number of projects in each period, maximum investment value along the entire horizon, maximum level of transmission losses, value of the penalty terms to be used if some constraints are violated.

- DEPSO settings - values for the parameters $p$, and $k_{\operatorname{Lam1,2}}$, $k_{\text {Lam } 3}, k_{c}$, number of particles in each population, maximum number of iterations, and maximum number of iterations without improvement of the fitness function before stopping.

- Results - the main result is the best expansion plan integrating a list of projects to be implemented along the planning horizon together with their placement along that horizon. A number of complementary results are also obtained as the value of the fitness function, decomposed in terms of investment and operational costs, the power flow in each branch in each period of the horizon, the losses in each period of the horizon and reliability results, in this case associated with the PNS values considering not only the state in which all components are available but also for the tested contingencies.

\subsection{Case 1 - Garver network}

\subsubsection{Single period analysis}

In an early development stage of the algorithm, we conducted numerous tests using the Garver Network with the main objective of validating the developed approach. For this reason, and also for simplicity, some of the features specified before were released:

- Losses - the losses were not included in this phase.

- PNS $(n-1)$ it was only considered PNS $(n)$ at this stage. Given the reduced size of the network, we considered that enforcing that $\operatorname{PNS}(n)$ is zero is enough and that $\operatorname{PNS}(n-1)$ reliability is meaningless.

- States and search space - when these tests were performed one was only reasoning in terms of $n p d+1$ states. The state corresponding to the postponement of projects was introduced latter.

In any case, these conditions do not alter the validity of the results obtained on testing DEPSO algorithm.

Table 2 presents the project list defined for the Garver network expansion problem. This list includes 17 projects, and it is based on the projects identified in several publications using the Garver network. For each possible branch in this list, it is specified the extreme buses, the resistance and reactive, the transmission capacity and the investment cost. The search space for a single period analysis has therefore $2^{17} \approx 1.31 \times 10^{5}$ positions.

The solution identified for the single period analysis of the Garver network coincides with the one reported in the literature: branch 3-5, 1 new line; branch 4-6, 3 new lines. Fig. 4 details the Garver network before and after running the expansion planning algorithm.

The algorithm was run 100 times with populations of $10,20,30$ and 50 particles. According to the results in Fig. 5, the performance of the developed DEPSO was remarkable, given that convergence was obtained for a very small number of iterations, even for populations of 10 particles. For populations with at least 20 particles in $98 \%$ of the cases the optimal solution indicated above was identified in less than 10 iterations. For populations with 50 particles, the optimal solution was identified in $100 \%$ of the runs showing the good convergence characteristics of the DEPSO.

The efficiency of DEPSO can be assessed by comparing the performance and the results obtained by other researchers for the same network adopting different methodologies. A comparison between DEPSO and GA can be made based on the study published in [46]. On this study it is presented a single period TEP on the Garver network, based on GA, and it is reported that usually about 21-30 
Table 1

Values adopted for several parameters of the DEPSO algorithm and for the TEP formulation.

\begin{tabular}{|c|c|c|}
\hline \multicolumn{2}{|l|}{ Parameter } & \multirow[t]{2}{*}{ Value } \\
\hline Symbol & Description & \\
\hline$p$ & Communication factor used in expression (15) & 0.3 \\
\hline$k_{\text {Lam } 1,2}$ & $\begin{array}{l}\text { Parameter used to activate the Lamarkian evolution step in } \\
\text { populations } 1 \text { and } 2\end{array}$ & 0.4 \\
\hline$k_{\text {Lam3 }}$ & $\begin{array}{l}\text { Parameter used to activate the Lamarkian evolution step in } \\
\text { population } 3\end{array}$ & 0.5 \\
\hline$k_{c}$ & $\begin{array}{l}\text { Parameter used to determine if the best global particle, bG } \\
\text { undergoes mutation or not, using (13) }\end{array}$ & 0.4 \\
\hline $\lg r$ & $\begin{array}{l}\text { Variation of the load from one period to the next one in } \\
\text { multiyear planning exercises (\%) }\end{array}$ & $\begin{array}{l}\text { Dependent on the network to be anaylsed. In the two test cases reported in this paper } 5 \% \\
\text { was used }\end{array}$ \\
\hline npd & Number of periods in the planning horizon & $\begin{array}{l}\text { In the two test cases reported in this paper, } 1 \text { was used in single period runs and } 4 \text { in } \\
\text { multiyear analysis }\end{array}$ \\
\hline$n p t$ & Number of particles in the population of the DEPSO & $\begin{array}{l}\text { Dependent on the run. Sections } 6.2 \text { and } 6.3 \text { report results obtained with } 10,20,30,50 \text {, } \\
80,100 \text { and } 150 \text { particles in each population }\end{array}$ \\
\hline npj & Number of projects in the list to be specified by the planner & $\begin{array}{l}\text { Dependent on the network. In the runs reported in this paper } 17 \text { projects were used for } \\
\text { the Garver network and } 28 \text { for the IEEE RTS network }\end{array}$ \\
\hline $\operatorname{pen}_{t} t=1 \ldots 6$ & Penalty terms used in expression (28) and (29) & $100,000 \$$ \\
\hline
\end{tabular}

iterations are required to get the results. Using the model and the solution algorithm reported in this paper fewer iterations are required to achieve the solution given that even with populations of 10 particles, only 11 iterations are needed to obtain the optimal solution in $100 \%$ of the runs, as showed in Fig. 5.

\subsubsection{Four period analysis}

The four period analysis also adopted the 17 projects listed in Table 1 . In this case, the search space has $5^{17} \approx 7.62 \times 10^{11}$ positions. This test was designed with the following assumptions: load increase of 5\% per period; fixed investment costs (for the sake of simplicity, it was considered that the effect of technological and market competitiveness outweighed the effect of inflation and capital discount rates); projects available from the year of entry into service; it should be underlined that the network configuration, and the corresponding power flows for subsequent years depend on previous year's configuration.

The best solution has an investment cost of $150 \times 10^{6} \$$ and it includes the following new branches:

- Period 1 - 1 branch 2-6, 1 branch 3-5, 1 branch 4-6 and 1 branch $1-5$.

- Period 2 - no new branches were scheduled for this period.

- Period 3 - 1 branch 4-6.

- Period 4 - 1 branch 3-5.

The evolution of the Garver network along the four periods is illustrated in Fig. 6. This figure shows the structure of the network from period 1 to period 4 , from the left to the right side.

As for the single period test, the algorithm was run 100 times with populations of $10,20,30,50$ and 80 particles. According to the results presented in Fig. 7, the 10 particles population apparently has a need for more iterations to converge. However, in $94 \%$ of cases the DEPSO reached the optimum in 100 iterations even using small populations with only 10 particles. Using populations of 30 particles one reaches the optimum in $95 \%$ of cases after 42 iterations, while with 80 particles similar results are found with only 20 iterations.

The performance of DEPSO can also be assessed in Fig. 8, which compares the results obtained using the EPSO reported in [55] and results obtained with DEPSO. This figure shows the frequency of identification of the best fitness for 25,50 and 100 iterations. In each test 100 runs were performed with $10,30,50$ and 100 particles. As it can be seen, DEPSO has better performance than the classical EPSO. Comparing the search spaces, in our case we have $5^{17}$ combinations and in the case of [55] there were $5^{15}$ possible combinations. Although the search space used by DEPSO is 25 times larger, the performance was improved given that DEPSO needs less iterations and smaller populations than EPSO to get the same results. In fact, in all the situations reported in Fig. 8 the percentage of identification of the best solution obtained for DEPSO is always larger than the one reported for EPSO. DEPSO is also able to escape from local minima, as it reaches $100 \%$ of optimal solutions in 100 iterations, independently from the population dimension.

\subsection{Case 2 - IEEE 24 bus reliability test system}

\subsubsection{Single period analysis}

The IEEE RTS network has 24 nodes, 35 lines and 32 generators. In order to obtain a more stressed network, and in line with other recent research works, the demand was set at $8550 \mathrm{MW}$ and the installed generation capacity is $10,215 \mathrm{MW}, 3$ times more than the original values, as adopted by many researchers.

The list of possible projects on the expansion planning exercise is shown in Table 3 in which the first four listed projects correspond to the installation of new transformers. As for Table 2, for each possible new branch, the extreme buses, the resistance, the reactance, the transmission capacity and the investment cost are specified. The search space has $3^{28} \approx 2.29 \times 10^{13}$ positions, for a single period analysis.

In the first place, the fitness considered the investment costs, no limit was imposed on the number of projects to be added, the quality of service was only evaluated for the particles having $\operatorname{PNS}(n)=0$ and the estimation of transmission losses was not included. Under these conditions, the best identified plan included two branches between nodes 1 and 5, one new branch $6-10$, two new branches between nodes 7 and 8 and one new branch 16-17. The corresponding investment cost was $1280 \mathrm{M} \$$. This solution compares favorably with the ones reported in [24] and in [34]. For instance, the best solution reported in [24] not considering transmission losses included one branch $6-10$, two branches $7-8$, one branch 10-12, one branch 11-13 and one branch $20-23$. Using the investment costs indicated in [24], the total investment cost associated with this solution is $170.29 \times 10^{6} \$$ while the total investment cost associated with the solution obtained by the DEPSO algorithm is $164.46 \times 10^{6} \$$.

In a second step, a new set of 20 tests, 1000 iterations each, with 10,30 and 100 particles were performed now including the estimate of transmission losses. The best solution ever found has an investment cost of $2150 \times 10^{6} \$$ and it includes two new transformers 
Table 2

Project list specified for the Garver network expansion problem.

\begin{tabular}{|c|c|c|c|c|c|c|}
\hline Branch no & From bus & To bus & Resist. (pu) & React. (pu) & Cap. (MW) & Cost $\left(10^{6} \$\right)$ \\
\hline 1 & 2 & 6 & 0.0800 & 0.0300 & 100 & 30 \\
\hline 2 & 2 & 6 & 0.0800 & 0.0300 & 100 & 30 \\
\hline 3 & 2 & 6 & 0.0800 & 0.0300 & 100 & 30 \\
\hline 4 & 2 & 4 & 0.1000 & 0.4000 & 100 & 40 \\
\hline 5 & 5 & 6 & 0.1476 & 0.6100 & 78 & 61 \\
\hline 6 & 3 & 5 & 0.0500 & 0.2000 & 100 & 20 \\
\hline 7 & 3 & 5 & 0.0500 & 0.2000 & 100 & 20 \\
\hline 8 & 3 & 5 & 0.0500 & 0.2000 & 100 & 20 \\
\hline 9 & 4 & 6 & 0.0800 & 0.3000 & 100 & 30 \\
\hline 10 & 4 & 6 & 0.0800 & 0.3000 & 100 & 30 \\
\hline 11 & 4 & 6 & 0.0800 & 0.3000 & 100 & 30 \\
\hline 12 & 4 & 6 & 0.0800 & 0.3000 & 100 & 30 \\
\hline 13 & 4 & 6 & 0.0800 & 0.3000 & 100 & 30 \\
\hline 14 & 1 & 4 & 0.1500 & 0.6000 & 80 & 60 \\
\hline 15 & 1 & 5 & 0.0500 & 0.2000 & 100 & 20 \\
\hline 16 & 1 & 2 & 0.1000 & 0.4000 & 100 & 40 \\
\hline 17 & 2 & 3 & 0.0500 & 0.2000 & 100 & 20 \\
\hline
\end{tabular}
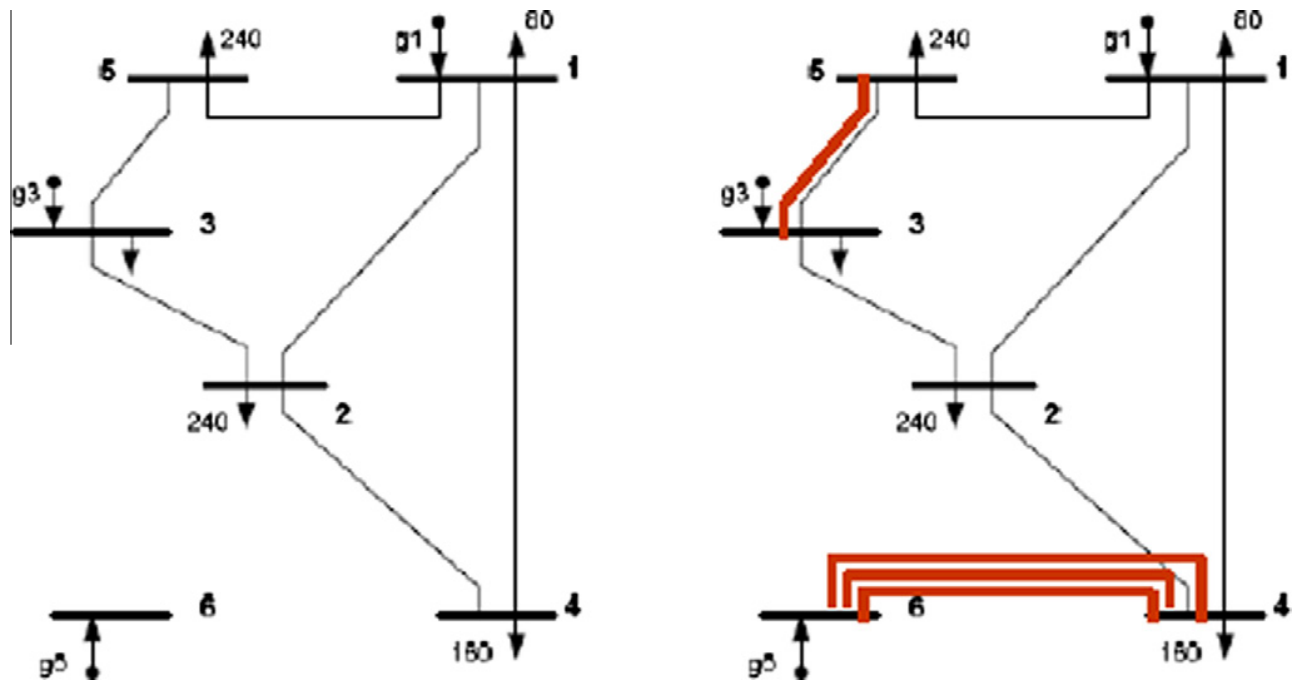

Fig. 4. Garver network before (left) and after the planning exercise (right).

$(9 / 11 ; 10 / 11)$ and three new lines $(2 / 4,6 / 10$ and $11 / 13)$. The most frequent investment value is $2160 \times 10^{6} \$$, and it corresponds to three different solutions: one solution with frequency $1 / 20$ (new lines $6 / 10 ; 7 / 8(2) ; 16 / 17(2) ; 20 / 23 ; 11 / 13)$, one solution with frequency $4 / 20$ (new lines: $2 / 4(2) ; 2 / 6 ; 6 / 10 ; 7 / 8(2) ; 16 / 19 ; 17 / 18$ ) and one solution with frequency $15 / 20$ (new transformer $9 / 11$ and new lines: $1 / 5 ; 6 / 10 ; 7 / 8(2) ; 20 / 23 ; 11 / 13)$.

With populations of 100 particles one reaches the fitness of $2160 \times 10^{6} \$$ in $100 \%$ of the runs and 600 iterations are enough to find the best solution in all runs. With populations of 30 particles

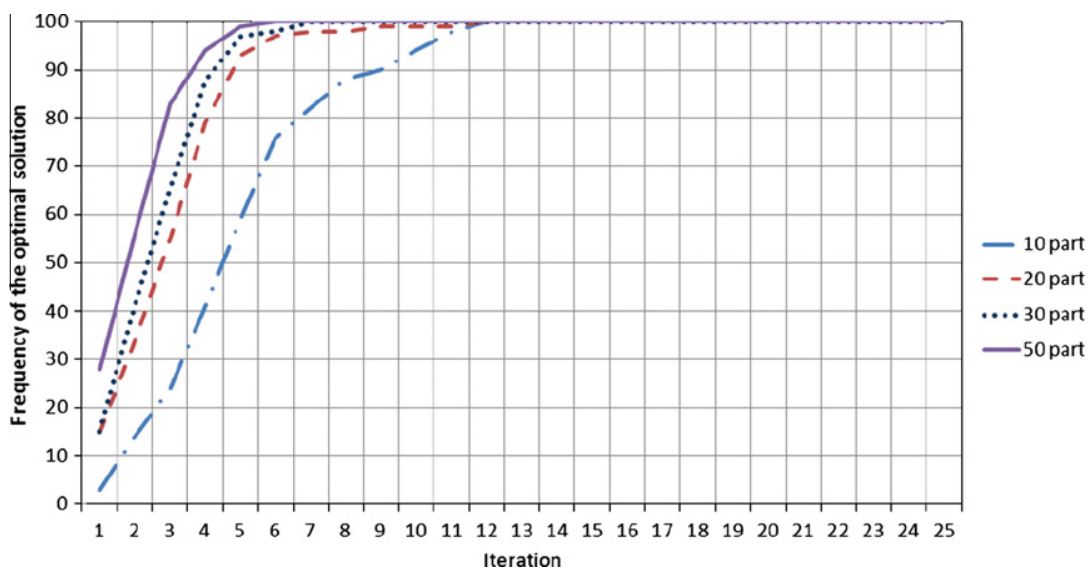

Fig. 5. DEPSO test with 10, 20, 30 and 50 particles (single period, Garver network). 

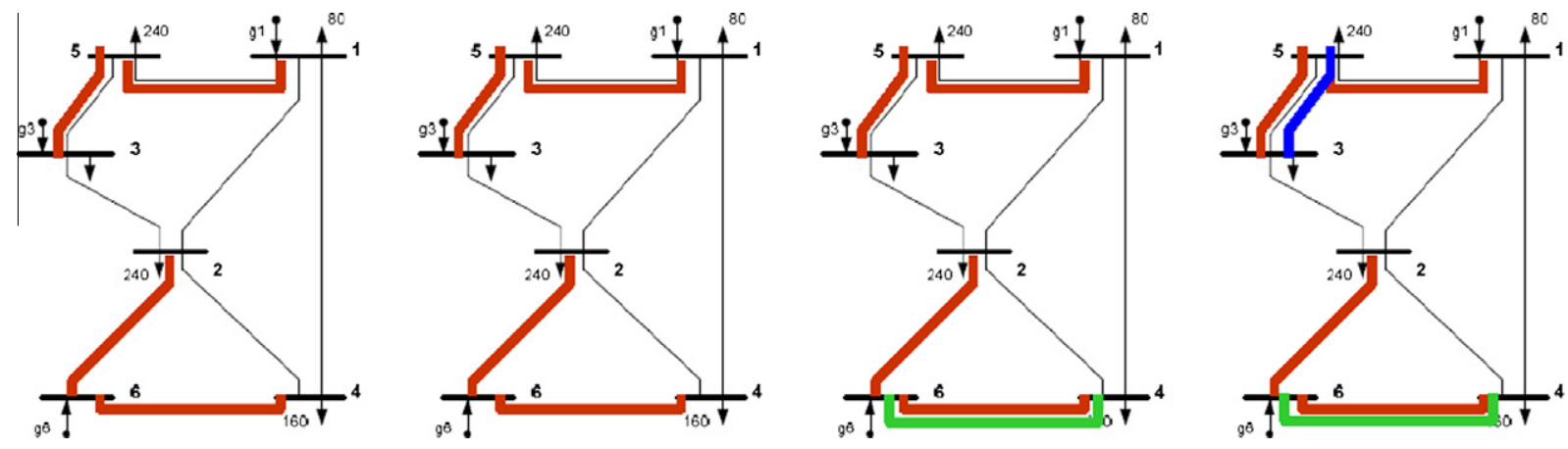

Fig. 6. Evolution of the Garver network from period 1 to period 4, from the left to the right side.

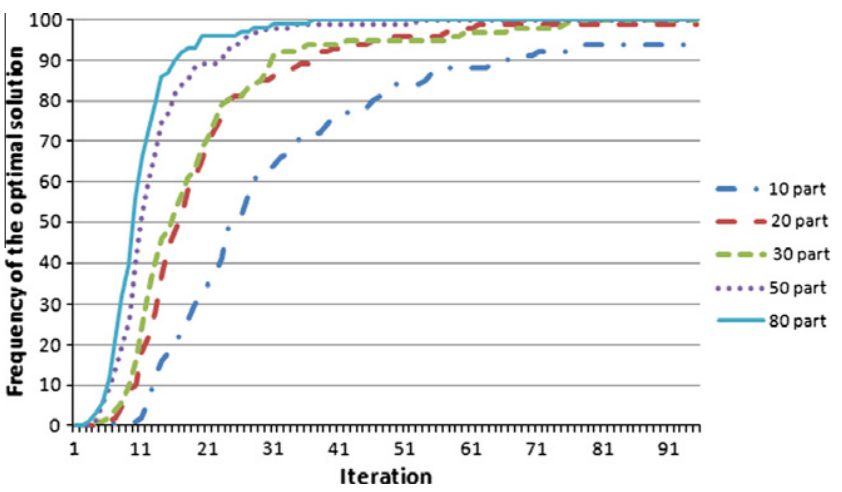

Fig. 7. DEPSO test with 10, 20, 30, 50 and 80 particles (multi period, Garver network).

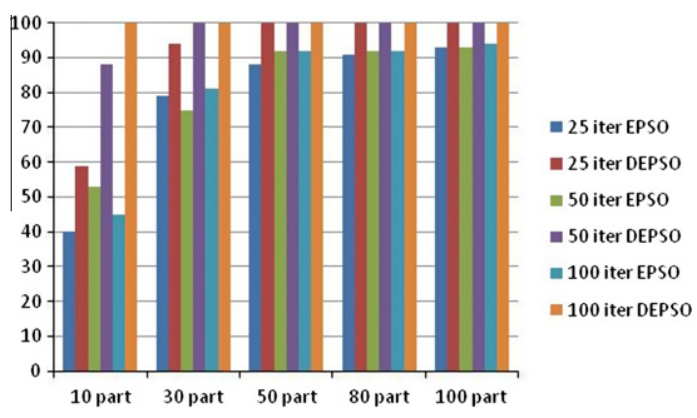

Fig. 8. DEPSO and EPSO performance comparison.

the success frequency was of $95 \%$ of the cases. One has to keep in mind that with 1000 iterations and a population of 30 particles, the DEPSO tests 90,000 solutions, in a search space with $3^{28} \approx 2.29 \times 10^{13}$ possible solutions.

\subsubsection{Four period analysis}

On the four period analysis it was adopted the same list of possible projects shown in Table 3. For these tests, and to cope with the demand increase of $5 \%$ a year, two new generators were added, in bus 4 (300 MW, similar to the generators in bus 7), and in bus 19 (591 MW, similar to the generator in bus 13 ). The search space for a four period analysis has $6^{28} \approx 6.14 \times 10^{21}$ positions.

Multi period tests were run with different sizes of the populations, namely with 30,100 and 150 particles and not considering the estimate of transmission losses. As can be seen in Fig. 9, and as expected, the performance of the run with 150 particles is much more interesting than those with 30 and 100 particles, not only because fewer iterations are needed to identify a solution, but also because the solution is slightly better given that the investment cost is more reduced.

Table 4 displays the best plans that were identified both considering the single period test and the multiperiod tests, using 30, 100 and 150 particles as indicated above. Regarding the single period analysis, the plan in Table 4 coincides with the one reported in Section 6.3.1 not considering the estimate of transmission losses. The columns in this table represent the associated investment cost and the projects that were included in the different solutions that were obtained in each test. For each of the four analyzed solutions, each project has a digit ranging from 0 to 5 . As detailed in the beginning of Section 5.3, a 0 means that such project was not included in this solution, a digit from 1 to 4 indicates the period in which the project is commissioned and 5 means that it was postponed. All the remaining projects listed in Table 3 and not present in Table 4 were not selected in any of these four solutions.

Comparing the results obtained for the multiperiod tests, one can see that the three solutions are similar in the sense that they include mostly the same projects. In particular the first and the second solutions differ only in the project $2 / 6$, which in the run with 100 particles is postponed from the second period to the fourth period. However, as indicated in Table 4, regarding the single period and the multi-period solutions one finds significant differences that are summarized below:

- The project $16 / 17$, identified in the single period solution, is only selected in the fourth period in one of the multi-period solutions.

- One of the projects $7 / 8$ is delayed from period 1 to period 2 .

- Several projects not elected in the singe period solution are included in the multi-period solution. This occurs with projects $3 / 24,10 / 12,2 / 6,11 / 13$ and $11 / 23$.

One may conclude that a multi-period analysis is not necessarily a combination of static schedules, and that not always the projects that are elected for a single period solution are considered in dynamic multi-period analysis. This means that transmission expansion planning exercises should be conducted in a dynamic and multiyear way so that the optimization problem preserves the holistic view over the network along the entire planning horizon. Among the three multi-period solutions, the one identified with 150 particles displays the most reduced value for the investment cost and should therefore be adopted, if no other information was available. In practice, however, TEP problems are very complex and in a real situation such a solution should be cross-validated considering other aspects, namely environmental ones. Eventually, the decision maker could also adopt a more costly solution as the ones obtained with populations with 30 or 100 particles. These two plans are equal regarding the first period, that is, they both include one branch $10 / 12$, one branch $1 / 5$, one branch $6 / 10$, one 
Table 3

Project list for the IEEE RTS network expansion problem.

\begin{tabular}{|c|c|c|c|c|c|c|}
\hline Branch no & From bus & To bus & Resist. (pu) & React. (pu) & Cap. (MW) & Cost $\left(10^{6} \$\right)$ \\
\hline 1 & 3 & 24 & 0.0000 & 0.4195 & 400 & 500 \\
\hline 2 & 9 & 11 & 0.0000 & 0.4195 & 400 & 500 \\
\hline 3 & 10 & 11 & 0.0000 & 0.4195 & 400 & 500 \\
\hline 4 & 10 & 12 & 0.0000 & 0.4195 & 400 & 500 \\
\hline 5 & 1 & 5 & 0.1090 & 0.4225 & 175 & 220 \\
\hline 6 & 1 & 5 & 0.1090 & 0.4225 & 175 & 220 \\
\hline 7 & 2 & 4 & 0.1640 & 0.6335 & 175 & 330 \\
\hline 8 & 2 & 4 & 0.1640 & 0.6335 & 175 & 330 \\
\hline 9 & 2 & 6 & 0.2485 & 0.9600 & 175 & 500 \\
\hline 10 & 2 & 6 & 0.2485 & 0.9600 & 175 & 500 \\
\hline 11 & 6 & 10 & 0.0695 & 0.3025 & 175 & 160 \\
\hline 12 & 7 & 8 & 0.0795 & 0.0032 & 175 & 160 \\
\hline 13 & 7 & 8 & 0.0795 & 0.0032 & 175 & 160 \\
\hline 14 & 8 & 10 & 0.2135 & 0.8255 & 175 & 430 \\
\hline 15 & 11 & 13 & 0.0305 & 0.2389 & 500 & 660 \\
\hline 16 & 12 & 13 & 0.0305 & 0.2380 & 500 & 660 \\
\hline 17 & 14 & 16 & 0.0250 & 0.1945 & 500 & 540 \\
\hline 18 & 15 & 21 & 0.0315 & 0.2450 & 500 & 680 \\
\hline 19 & 15 & 24 & 0.0335 & 0.2595 & 500 & 720 \\
\hline 20 & 16 & 17 & 0.0165 & 0.1295 & 500 & 360 \\
\hline 21 & 16 & 17 & 0.0165 & 0.1295 & 500 & 360 \\
\hline 22 & 16 & 19 & 0.0150 & 0.1150 & 500 & 320 \\
\hline 23 & 17 & 18 & 0.0090 & 0.0720 & 500 & 200 \\
\hline 24 & 20 & 23 & 0.0140 & 0.1080 & 500 & 300 \\
\hline 25 & 11 & 13 & 0.0305 & 0.2380 & 500 & 660 \\
\hline 26 & 12 & 13 & 0.0305 & 0.2380 & 500 & 660 \\
\hline 27 & 11 & 14 & 0.0305 & 0.2380 & 500 & 580 \\
\hline 28 & 14 & 16 & 0.0250 & 0.1945 & 500 & 540 \\
\hline
\end{tabular}

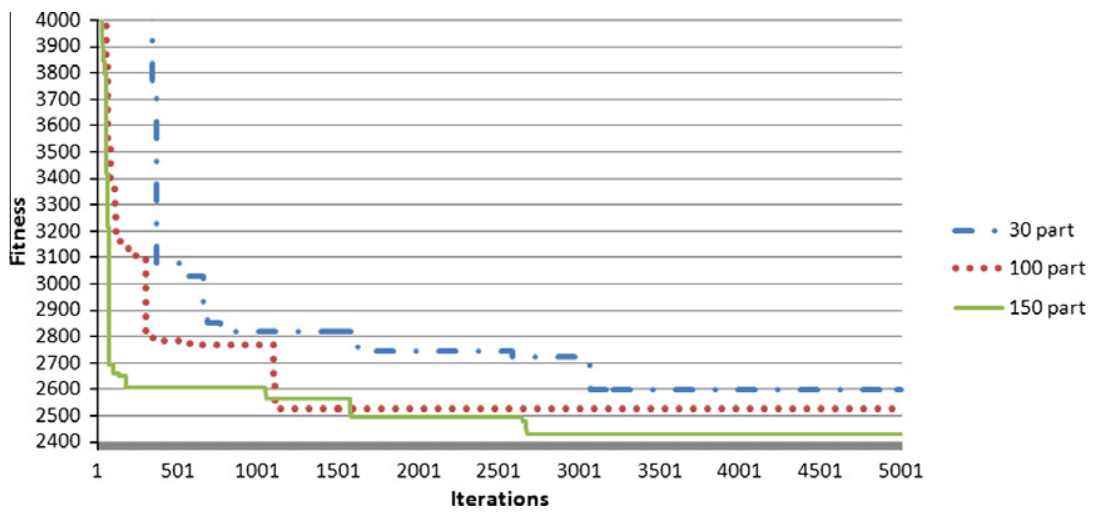

Fig. 9. Evolution of the fitness function for a deterministic multi-period test with 30,100 and 150 particles.

branch $7 / 8$ and one branch $11 / 13$ which means they imply no different decisions till period 2 . In a more conservative decision making environment, this could be interpreted as a way of postponing decisions that would start differentiating the expansion plans. This could constitute an argument to favor the solutions obtained with populations with 30 or 100 particles, in detriment of the plan obtained with a 150 particle population because this one would require taking differentiating and eventually more risky decisions right from the beginning of the planning horizon.

\subsubsection{Four period analysis considering demand uncertainties}

When considering uncertainties on the four period analysis, the same list of possible projects on the expansion planning shown in Table 3 was adopted. The uncertainty in each load is modeled using a fuzzy number with a triangular membership function. Each particle is tested in five points of the membership function: $95 \%$, $97.5 \%, 100 \%, 102.5 \%, 105 \%$ of the central value of each load. It should be noted that point of $100 \%$ corresponds to the deterministic solution, already tested in the previous section. All particles were tested by assessing the $\operatorname{PNS}(n-1)$ with the fuzzy load.

From the results we observe the following:

- The solutions which have identified the inclusion of a transformer 3/24 display poor quality in the presence of load uncertainties.

- Some solutions that have identified the exclusion of certain lines (such as $1 / 5$ and $11 / 23$, which interconnect generating buses with relevant loads) also show poor quality, being very sensitive to load variations.

- Only $19 \%$ of the 100 solutions that integrate the final population failed the load uncertainty test.

- The 34 particles with better fitness showed no problem in accommodating load uncertainties.

- There is no correlation between the number of projects and network behavior, as it was observed that solutions with fewer projects and better fitness have better performance than solutions with more projects and worse fitness, namely solutions having larger investment cost. 
Table 4

Deterministic expansion plans for the single and multi-period analysis not considering the estimate of transmission losses.

\begin{tabular}{|c|c|c|c|c|c|c|c|c|c|c|c|c|c|}
\hline \multirow[t]{2}{*}{ Solutions } & \multirow[t]{2}{*}{ Investment cost $\left(10^{6} \$\right)$} & \multicolumn{12}{|c|}{ Expansion projects from the list in Table 3} \\
\hline & & $3-24$ & $10-12$ & $1-5$ & $1-5$ & $2-6$ & $6-10$ & $7-8$ & $7-8$ & $11-13$ & $11-13$ & $16-17$ & $11-23$ \\
\hline \multicolumn{14}{|c|}{4 Period analysis } \\
\hline 30 Part & 2599.16 & 0 & 1 & 1 & 5 & 2 & 1 & 1 & 2 & 1 & 3 & 5 & 5 \\
\hline 100 Part & 2527.44 & 0 & 1 & 1 & 5 & 4 & 1 & 1 & 2 & 1 & 3 & 0 & 0 \\
\hline 150 Part & 2427.72 & 4 & 1 & 1 & 5 & 0 & 1 & 1 & 2 & 0 & 0 & 4 & 1 \\
\hline \multicolumn{14}{|c|}{1 Period analysis } \\
\hline 30 Part & 1280.00 & 0 & 0 & 1 & 1 & 0 & 1 & 1 & 1 & 0 & 0 & 1 & 0 \\
\hline
\end{tabular}

This information can thus be used to characterize in a more complete way each plan in the final population so that the planner has more insight on the behavior on the network in view of the specified load uncertainties. This means he can select the final plan in a more robust and less risky way, not just taking into account the investment cost associated with each possible solution.

\section{Conclusions}

This paper described a multiyear transmission expansion planning formulation having mixed integer nature. This problem was solved using a discrete version of the EPSO algorithm, termed as DEPSO. Regarding the TEP formulation it is important to mention that it has a dynamic multiyear nature so that all periods in the planning horizon are treated in the same run of the planning exercise. This means that the model can profit from an holistic view on the problem, instead of obtaining separate solutions for consecutive years. On the other hand, the developed model incorporates a number of constraints having technical, financial and reliability natures, which are very much in line with the concerns of system operators. In fact, expansion plans should comply with physical operation laws of power systems (related with power flow constraints), and TSO's often mention financial limitations for instance associated with the number of projects that can be built simultaneously. It is still important to mention that the grid codes governing the activity of several TSO's impose that transmission systems should be planned and operated so that they are secure regarding $n-1$ and, in some cases, $n-2$ contingencies. These constraints are easily incorporated in the developed approach since each candidate plan identified by the DEPSO is analyzed against contingencies and if power not supplied arises, then the corresponding fitness function is penalized. The developed approach also admits that the loads are modeled by triangular fuzzy numbers as a way to incorporate the uncertainty that can affect them. This ultimately means that the final plan should be adequate regarding the most credible load scenario associated with the central values of the load triangular numbers but should also display an adequate behavior if the load changes. Incorporating load uncertainties in this way means that the expansion plans will be more robust in the sense that they will still be adequate despite the demand is affected by uncertainties along the horizon. The analysis with load uncertainty also helps on identifying the most critical branches, those which reveal a greater influence on PNS. This knowledge can then be useful in order to expand the dynamic TEP project list with new projects that can address specific bottlenecks identified in the system.

The TEP problem was solved using the developed DEPSO algorithm. The DEPSO algorithm was tested using different networks and it showed a very good performance namely in terms of converging to the optimal solutions. On the other hand, when compared with other heuristics as EPSO, the convergence took fewer iterations and a greater ability to escape from local minima was observed. It is still important to notice that the diversity in the population is guaranteed, which results in a variety of solutions, among which the planner can choose the one that best suits his needs. The results obtained with the DEPSO algorithm were compared with the results reported by other researchers to the same networks and the solutions provided by DEPSO compare very favorably with them, namely regarding the total investment cost.

Research work is now going on in order to test the DEPSO algorithm and the developed TEP model on realistic transmission systems, so that new reports on the near future are expected on this topic.

\section{References}

[1] Stoft S. Power system economics: designing markets for electricity. New York: IEEE Press \& Wiley Interscience, A John Wiley \& Sons Inc. Publication; 2002.

[2] Pereira AJC, Saraiva JT. Generation Expansion Planning (GEP): a long-term approach using system dynamics and Genetic Algorithms (GAs). Energy Int J 2011;36:5180-99.

[3] Rocha MJC, Saraiva JT. Muliyear transmission expansion planning using discrete evolutionary particle swarn optimization. In: Proc 17th power systems computation conf, Stockholm; 2011.

[4] EU Parliament and Council. Directive 2009/72/EC of the EU Parliament and Council of 13 July 2009 establishing rules for the internal electricity market Brussels; 2009.

[5] Cedeno EB, Arora S. Performance comparison of transmission network expansion planning under deterministic and uncertain conditions. IJEPES 2011;33:1288-95.

[6] Gomes BA, Saraiva JT. Addressing the impact of demand and generation cost uncertainties in the operation of power systems. In: Proc of the IFAC international symposium on power plants and power systems control, Toulouse; 2012.

[7] Pereira MV, Pinto LMVG, Cunha SH, Oliveira GC. A decomposition approach to automated generation/transmission expansion planning. IEEE Trans Power Apparat Syst 1985;PAS-104:3074-83.

[8] Moon GH, Joo SK, Hur D, Jeong HS, Ryu HS, Cho KW. Stochastic integrated generation and transmission planning method with Gradient Radar Step (GRS). In: Proc 2009 Asia-Pacific power and energy engineering conference, APPEEC 2009, Seoul; 2009. p. 1-4.

[9] Saboori H, Mohammadi M, Tahe R. Composite generation and transmission expansion planning considering the impact of wind power penetration. In: Proc 2011 Asia-Pacific power and energy engineering conf, APPEEC 2011, Wuhan; 2011. p. 1-6.

[10] Unsihuay-Vila C, Lima JWM, Souza ACZ, Perez-Arriaga IJ. Multistage expansion planning of generation and interconnections with sustainable energy development: a multi-objective model. IJEPES 2011;33:258-70.

[11] Akbari T, Rahimi-Kian A, Bina MT. Security constrained transmission expansion planning: a stochastic multiobjective approach. IJEPES 2012;43:444-53.

[12] Latorre G, Cruz R, Areiza J, Villegas A. Classification of publications and models on transmission expansion planning. IEEE Trans Power Syst 2003;18:938-46.

[13] Lee CW, Ng SKK, Zhong J, Wu FF. Transmission expansion planning from past to future. In: Proc 2006 IEEE PES power systems conf and exposition, PSCE 2006, Atlanta; 2006. p. 257-65.

[14] Fonseka J, Miranda V. A hybrid meta-heuristic algorithm for transmission expansion planning. Int J Comput Math Electr Electron Eng 2004;23:250-62.

[15] Sadegheih A, Drake P. System network planning expansion using mathematical programming, genetic algorithms and Tabu search. Energy Convers Manage 2008;49:1557-66.

[16] Gajbhiye RK. An expert system approach for multi-year short term transmission expansion planning: an Indian experience. IEEE Trans Power Syst 2008;23:226-37.

[17] Kaltenbach JC, Peshon J, Gehrig EH. A mathematical optimization technique for the expansion of electrical power transmission systems. IEEE Trans Power Apparat Syst 1970;PAS-89:113-19.

[18] Dusonchet YP, El Abiad AH. transmission planning using discrete dynamic optimization. IEEE Trans Power Apparat Syst 1973;PAS-92:1358-71.

[19] Torre S, Conejo A, Contreras J. Transmission expansion planning in electricity markets. IEEE Trans Power Syst 2008;23:238-48. 
[20] Hashimoto SHM, Romero R, Mantovani JRS. Efficient linear programming algorithm for the transmission network expansion planning problem. IEE Proc Gen Trans Dist 2003;150:536-42.

[21] El-Metwally M, Harb A. Transmission planning using admittance approach and quadratic programming. Electr Mach Power Syst 1993;21:69-83.

[22] Sanchez IG, Romero R, Mantovani JRS, Rider MJ. Transmission expansion planning using the dc model and non-linear programming technique. IEE Proc Gen Trans Dist 2005;152:763-69.

[23] El-Abiad AH, Dusonchet YP. Discrete optimization and the planning of electric power networks. IEEE Trans Circuit Theory 1973;20:230-8.

[24] Alguacil N, Motto A, Conejo A. Transmission expansion planning: a mixed integer LP approach. IEEE Trans Power Syst 2003;18:1070-7.

[25] Haffner S, Monticelli A, Garcia A, Mantovani J, Romero R. Branch and bound algorithm for transmission systems expansion planning using a transportation model. IEE Proc Gen Trans Dist 2000;147:149-56.

[26] Binato S, Pereira MV, Granville S. A new benders decomposition approach to solve power transmission network design problems. IEEE Trans Power Syst 2001;16:235-40.

[27] Romero R, Monticelli A. A hierarchical decomposition approach for transmission network expansion planning. IEEE Trans Power Syst 1994;9:373-80.

[28] Gallego RA, Alves AB, Monticelli A, Romero R. Parallel simulated annealing applied to long term transmission network expansion planning. IEEE Trans Power Syst 1997;12:181-8.

[29] Braga AS, Saraiva JT. A multiyear dynamic approach for transmission expansion planning and long-term marginal costs computation. IEEE Trans Power Syst 2005;20:1631-9.

[30] Leite da Silva AM, Manso LA, Resende LC, Rezende LS. Tabu search applied to transmission planning considering losses and interruption costs. In: Proc 10th PMAPS conf, int conf probabilistic methods applied to power systems, Puerto Rico; 2008.

[31] Binato S, Oliveira GC, Araújo JL. A greedy randomized adaptive search procedure for transmission expansion planning. IEEE Trans Power Syst 2001;16:247-53.

[32] Leou RC. A multiyear transmission planning under deregulated market. IJEPES 2011;33:708-14

[33] Silva IJ, Rider MJ, Romero R, Garcia A, Murari CA. Transmission network expansion planning with security constraints. IEE Proc Gen Trans Dist 2005;152:828-36.

[34] Romero R, Rider MJ, Silva IJ. A metaheuristic to solve the transmission expansion planning. IEEE Trans Power Syst 2007;22:2289-91.

[35] Leite da Silva AM, Resende LS, Honório LM, Manso LAF. Performance comparison of metaheuristics to solve the multi stage transmission expansion planning problem. IET Proc Gen Trans Dist 2011;5:360-7.

[36] Rezende LS, Leite da Silva AM, Honório LM. Artificial immune system applied to the multi stage transmission expansion planning. Springer Lect Notes Comput Sci 2009;5666:178-91.

[37] Sensarma PS, Rahmani M, Carvalho A. A comprehensive method for optimal expansion planning using particle swarm optimization. In: Proc IEEE power engineering society winter meeting, vol. 2; 2002. p. 1317-22.
[38] Leite da Silva AM, Rezende LS, Manso LA, Anders GJ. Transmission expansion planning: a discussion on reliability and $N-1$ security criteria. In: Proc 11 th PMAPS conf, int conf probabilistic methods applied to power systems, Singapore; 2010.

39] Alvarez J, Ponnambalam K, Quintana VH. Transmission expansion under risk using stochastic programming. In: Proc 9th PMAPS conf, int conf probabilistic methods applied to power systems, Stockholm; 2006.

[40] Braga AS, Saraiva JT. Long term marginal prices - solving the revenue reconciliation problem of transmission providers. In: Proc 15 th power systems computation conf, Liege; 2005.

[41] Zadeh LA. Fuzzy sets. Inf Control 1965;8:338-53.

[42] Kennedy J, Eberhart RC. Particle swarm optimization. In: Proc 1995 IEEE int conf neural networks; 1942-1948.

[43] Miranda V, Fonseca N. EPSO - evolutionary particle swarm optimization, a new algorithm with applications in power systems. In: Proc 2002 IEEE/PES Asia Pacific transmission and distribution conference and exhibition, vol. 2; 2002. p. 745-50

[44] Miranda V, Keko H, Jararillo A. Stochastic star communication topology in evolutionary particle swarms (EPSO). Int J Comput Intell Res 2008:4:105-16.

[45] Kennedy J, Eberhart R. A discrete binary version of the particle swarm optimization algorithm. In: Proc 1997 conf on systems, man, and, cybernetics 1997. p. 4104-09.

[46] Gallego RA, Monticelli A, Romero R. transmission system expansion planning by an extended genetic algorithm. IEE Proc Gen Trans Dist 1998;145:329-35.

[47] Jin Y, Cheng H, Yan J, Zhang L. New discrete method for particle swarm optimisation and its application in transmission network expansion planning. Electr Power Syst Res 2007;77:227-33.

[48] Caponetto R, Fortuna L, Fazzino S, Xibilia M. Chaotic sequences to improve the performance of evolutionary algorithms. IEEE Trans Evol Comput 2003;7:289-304.

[49] Malik R, Rahman T, Hashim S, Ngah R. New particle swarm optimizer with sigmoid increasing inertia weight. Int J Comput Sci Secur 2007;1:35-44.

[50] Houck C, Joines J, Kay M, Wilson J. Empirical investigation of the benefits of partial Lamarkianism. Evol Comput 1997;5:31-60.

[51] Rocha MJC. Transmission expansion planning - a multiyear approach considering uncertainties. PhD thesis. Faculdade de Engenharia da Universidade do Porto; 2012.

[52] Garver L. Transmission network estimation using linear programming. IEEE Trans Power Apparat Syst 1970;PAS-89:1688-97.

[53] IEEE Reliability Test System-1996. IEEE Trans Power Apparat Syst 1979;PAS98:2047-54.

[54] Rezende LS, Leite da Silva AM, Honório LM. artificial immune systems and differential evolution based approaches applied to multi-stage transmission expansion planning. In: Proc 15th int conf on intelligent systems, ISAP 2009 Curitiba; 2009. p. 1-6.

[55] Pringles RM, Miranda V, Garcés F. Optimal expansion of the transmission system using EPSO. In: Proc VII Latin American congress on electricity \& transmission, Valparaiso, Paper C074; 2007. 